\title{
Theoretical Analysis of Mass Transfer in a Droplet Moving Down in Supercritical Carbon Dioxide Environment
}

\author{
Rahul Kumar, Hari Mahalingam, and Krishna K. Tiwari
}

\begin{abstract}
Supercritical antisolvent crystallization processes (SAS) exploits the unique properties of supercritical fluid to produce the micron size particles of pharmaceutical compounds. In this work, rifampicin which is an antibiotic drug mainly used for treatment of tuberculosis is considered as model drug. A thermodynamic model has been used to predict the solubility of rifampicin in the mixture of carbon dioxide and dimethyl sulfoxide. Peng-Robinson equation of state has been used to calculate the molar volume of binary mixture. All the model equations have been solved in MATLAB 7.1. It is observed that initial droplet diameter depends on flow rate and nozzle diameter. Mass transfer between this droplet and supercritical carbon dioxide has been studied and droplet diameter is calculated as it moves down. Effect of process parameters such as flow rate, nozzle diameter, pressure and temperature have been studied
\end{abstract}

Index Terms-Supercritical, antisolvent, droplet, rifampicin, crystallization.

\section{INTRODUCTION}

Fine particles of pharmaceutical compounds are in great demand for various reasons such as use of more suitable drug delivery paths in the body, less amount of required dosage of drug as the efficiency of fine drug particles are higher and increase in the bioavailability of drug in the body. Conventional micronization processes such as jet milling and spray drying can result in wide size distribution, thermal denaturing, excessive surface change or roughness [1]. Supercritical fluid base micronization processes have shown an advantage over these traditional processes by overcoming these disadvantages. The advantages of supercritical fluid base processes include high purity of products, control of crystal polymorphism and possibility of processing thermolabile molecules, a single step process and easy downstream processing and environmentally acceptable technology [2].

A supercritical fluid is defined as a substance above its critical temperature and critical pressure. The physical properties of SCF vary between gas- and liquid- like characteristic [3]. Supercritical fluid can be used as solvent or antisolvent in the micronization process. Carbon dioxide $\left(\mathrm{CO}_{2}\right)$ is used extensively as a supercritical fluid due to its desirable properties such as relatively accessible critical

Manuscript received April 10, 2014; revised July 21, 2014.

The authors are with the Department of Chemical Engineering, Jaypee University of Engineering \& Technology, Guna- 473226, India (corresponding author: Rahul Kumar; tel.: +91-7544-267310; fax: +917544-267011; e-mail: rahul.kumar@juet.ac.in, rahul.iitrahul@gmail.com, hari.mahalingam@juet.ac.in, kk.tiwari@juet.ac.in). point at temperature $31.1^{\circ} \mathrm{C}$ and pressure at 73.8 bar, abundance and its low toxicity [4]. The particle formation process involving $\mathrm{CO}_{2}$ as a solvent and antisolvent are referred as Rapid Expansion of Supercritical Solution (RESS) and Supercritical Antisolvent Process (SAS) respectively. The low solubility of the majority of drugs in carbon dioxide limits its use as a pure solvent [5] and therefore role of antisolvent of supercritical fluid $\left(\mathrm{CO}_{2}\right)$ has been preferred to produce fine drug powders.

In Supercritical antisolvent process, drug of interest is first dissolved into an organic solvent and this solution is injected into supercritical fluid environment which acts as an antisolvent. The solubility of drug in solvent decreases due to diffusion of antisolvent which causes supersaturation and subsequently crystallization take places. A high and rapid supersaturation is induced in the solution which yield small size particle with narrow size distribution. A variety of pharmaceutical compounds such as salbutamol [6], atenolol [7], theophylline [8], cefonicid [9], erlotinib hydrochloride and fulvestrant [10], ampicillin [11], cilostazol [12], cyclodextrins [13] have been processed using supercritical antisolvent micronization process and effect of process parameters such as temperature, pressure, flow rate of solution, solute concentration have been studied.

SAS process has been successful for the producing spherical morphology of particles which has large possibility of industrial application because spherical microparticles in the range of $1-5 \mu \mathrm{m}$ are very useful in the pharmaceutical field for aerosol formulations [14]. A number of biopolymers and lysozyme also have been successfully micronized [15], [16] via supercritical antisolvent process.

Solubility of drug particle to be micronized plays a significant role on the mechanism of particle formation. Rossmann et al. [17] found that SAS system $\mathrm{YAc} / \mathrm{DMSO} / \mathrm{CO}_{2}$, which is composed of yttrium acetate (YAc) as solute, dimethyl sulfoxide (DMSO) as solvent, and carbon dioxide $\left(\mathrm{CO}_{2}\right)$ as antisolvent, precipitates amorphous particles. The SAS system $\mathrm{PCM} / \mathrm{EtOH} / \mathrm{CO}_{2}$, which is composed of paracetamol (PCM) as solute, ethanol (EtOH) as solvent and $\mathrm{CO}_{2}$ as antisolvent, crystalizes crystal. These different mechanisms of particle formation have been correlated with the saturation solubility of the solutes which found to be approximately four orders of magnitude larger for the crystallizing system than for the precipitating system.

Experimental data for solubility of acetaminophen in the ternary system of $\mathrm{CO}_{2} /$ ethanol/acetaminophen has been reported by Wubbolts et al. [18]. This solubility data was correlated with Peng-Robinson equation of state. The 
equations of state parameters were evaluated fitting the experimental data. Kikic et al. proposed estimation method based on fugacity concept and determined the solubility of drugs such as acetaminophen, acyclovir, atenolol, carbamazepine, ibuprofen, naproxen, nimesulide and sotatlol hydrochloride in the mixture of $\mathrm{CO}_{2}$ and organic solvents (ethanol, dimethylsulfoxide, acetone) at a constant temperature and a variable pressure.

In this work, system considered is rifampicin/DMSO/CO 2 . As carbon dioxide diffuses into the solution droplet containing rifampicin and DMSO, the volume of solvent changes. The partial molar volume and subsequently total volume of solvent is calculated. The solubility of rifampicin has been calculated assuming mole fraction of solid solute in the solution is proportional to total volume of solvent in the mixture of carbon dioxide and solvent in the droplet.

\section{Model EQUATIONS}

Supercritical antisolvent micronization process starts with the atomization of solution containing a solute to be micronized dissolved in organic solvent in supercritical fluid environment. In this work solubility of rifampicin is to be determined in the ternary system of rifampicin/DMSO/CO As the solution of rifampicin $\mathrm{n}$ dimethyl sulfoxide is atomized through a nozzle in $\mathrm{SC} \mathrm{CO}_{2}$, droplets are formed. This droplet is a binary mixture at the tip of the nozzle. As droplet moves down in $\mathrm{SC} \mathrm{CO}_{2}$ environment, two way mass transfer from droplet to $\mathrm{SC} \mathrm{CO}_{2}$ environment and $\mathrm{SC} \mathrm{CO}_{2}$ to droplet- take place. Rifampicin is insoluble in $\mathrm{SC} \mathrm{CO}_{2}$ and with diffusion of $\mathrm{SC} \mathrm{CO}_{2}$ in droplet, solvent power in ternary mixture decreases which leads a reduction in the solubility of rifampicin. When this solubility becomes higher than actual amount of rifampicin in the droplet, later becomes saturated and precipitation of rifampicin starts.

The droplets produced by the nozzle in supercritical antisolvent process are small, in the order of 50-100 $\mu \mathrm{m}$ [19]. In this work, droplet size is calculated using the equation cited by Rahul et al. [20]. This is also assumed that droplet is formed at the tip of the nozzle and velocity of the droplet is calculated applying continuity equation at the nozzle. Droplet moves downward in the precipitator. The journey of droplet is discretized into time interval $(=0.001 \mathrm{~s})$ and mass transfer of $\mathrm{CO}_{2}$ into droplet and DMSO from droplet is calculated.

The density of droplet is calculated using Peng-Robinson equation:

$$
\begin{aligned}
P=\frac{R T}{v-b_{m}}- & \frac{a_{m}}{v\left(v+b_{m}\right)+b_{m}\left(v-b_{m}\right)} \\
b & =0.0778 \frac{R T_{c}}{P_{c}} \\
a & =a_{T_{c}} \alpha\left(T_{r}, \omega\right) \\
a_{T_{c}} & =0.45724 \frac{R^{2} T_{c}^{2}}{P_{c}}
\end{aligned}
$$

$$
\begin{gathered}
\alpha=\left[1+\beta\left(1-T_{R}^{\frac{1}{2}}\right)\right]^{2} \\
\beta=0.37464+1.54226 \omega-0.2699 \omega^{2} \\
a_{m}=\sum_{i} \sum_{j} x_{i} x_{j} a_{i j} \\
a_{i j}=\left(a_{i i} a_{j j}\right)^{1 / 2}\left(1-k_{i j}\right) \\
b_{m}=\sum_{i} \sum_{j} x_{i} x_{j} b_{i j} \\
b_{i j}=\frac{\left(b_{i i}+b_{j j}\right)}{2}\left(1-l_{i j}\right)
\end{gathered}
$$

This equation is can be written as a cubic equation of state in the following form:

$$
\begin{gathered}
Z^{3}-(1-B) Z^{2}+\left(A-3 B^{2}-2 B\right) Z-\left(A B-B^{2}-B^{3}\right) \\
=0
\end{gathered}
$$

where

$$
\begin{gathered}
A=\frac{a P}{R^{2} T^{2}} \\
Z=\frac{P v}{\mathrm{RT}} \\
B=\frac{b P}{R T}
\end{gathered}
$$

This cubic equation of state has been solved using Newton -Rapson method taking ideal gas volume as first guess.

$$
\begin{gathered}
v(1)=\frac{R T}{P} \\
Z(2)=Z(1)-\frac{f[z(1)]}{f^{\prime}[z(1)]} \\
Z(n+1)=Z(n)-\frac{f[z(n)]}{f^{\prime}[z(n)]}
\end{gathered}
$$

At $t=0$, droplet is just formed. At $t=0+\boldsymbol{\Delta} t$, droplet moves downward and supercritical carbon dioxide diffuses into the droplet and in this same time interval mass transfer solvent dimethyl sulfoxide also take place from droplet to precipitator. The mass transfer of carbon dioxide and dimethyl sulfoxide is calculated using following relation:

$$
N_{1}=k_{L}\left(C_{L, 1}^{i}-C_{L, 1}\right)=k_{L}\left(\rho_{L}^{i} x_{1}^{i}-\rho_{L} x_{1}\right)
$$

where $N_{1}$ is molar flux of carbon dioxide into droplet

$$
N_{2}=k_{G}\left(C_{G, 2}^{i}-C_{G, 2}\right)=k_{G}\left(\rho_{G}^{i} y_{2}^{i}-\rho_{G} y_{2}\right)
$$


where $N_{2}$ is the molar flux of organic solvent dimethyl sulfoxide into precipitator having supercritical carbon dioxide medium.

Total moles of droplet can be calculated once $N_{l}$ and $N_{2}$ are calculated and subsequently mole fraction carbon dioxide and dimethyl sulfoxide can be calculated in the droplet. With the help of mole fraction of carbon dioxide in the droplet, equilibrium solubility of rifampicin is calculated using following formula [21]

$$
x_{3}^{*}(T, P)=\frac{\left(1-x_{1}\right) v_{2}\left(T, P, x_{1}\right) / v\left(P, x_{1}\right)}{\left(1-x_{10}\right) v_{2}\left(T, P, x_{10}\right) / v\left(P_{0}, x_{10}\right)}\left(x_{30}^{*}\left(T, P_{0}\right)\right)
$$

$v_{2}$ is the partial molar volume of dimethyl sulfoxide which is calculated using Pen-Robinson equation.

Droplet then moves to next interval and all these calculations such as density, mass flow rate of $\mathrm{CO}_{2}$ and solvent, partial molar volume and solubility are done in MATLAB 7.1 for next interval. Journey of droplet in the precipitator discretized till all the solvent evaporated from the droplet.

\section{RESUlTS AND DISCUSSION}

Supercritical antisolvent process starts with the atomization of solution in supercritical medium. From Fig.1 it can be concluded that droplet diameter increases first and then decreases. Droplet diameter depends on the total number of moles present in the droplet. As droplet moves downward, $\mathrm{CO}_{2}$ diffuses rapidly into droplet while dimethyl sulfoxide evaporates from droplet. But net mass transfer is into droplet due to high diffusivity of $\mathrm{CO}_{2}$ than dimethyl sulfoxide. Therefore the total number of moles in the droplet increases and its diameter increases.

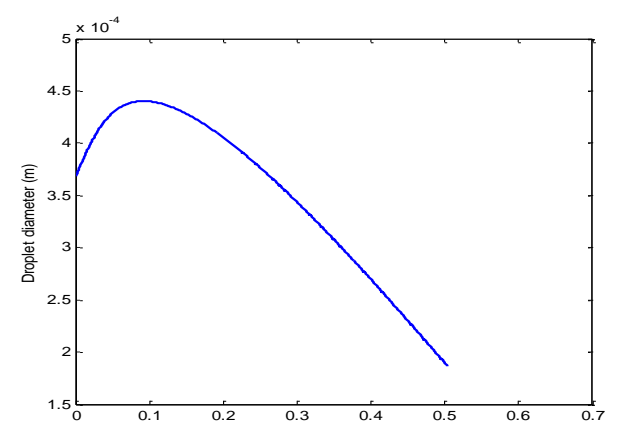

Fig. 1. Droplet diameter v/s time at $T=313 \mathrm{~K}, P=80 \mathrm{bar}, d_{n}=100 \mu \mathrm{m}, F$ $=60 \mathrm{ml} / \mathrm{h}$.

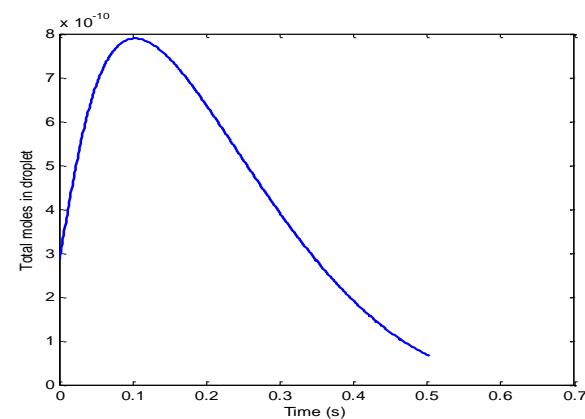

Fig. 2. Total number of moles v/s Time (s) at $T=313 \mathrm{~K}, P=80 \mathrm{bar}, d_{n}=$ $100 \mu \mathrm{m}, F=60 \mathrm{ml} / \mathrm{h}$.
Total moles in droplet are varying with the time are shown in Fig. 2. This is found that total moles decreases due to decrease in the moles of $\mathrm{CO}_{2}$ in the droplet. This occurs because concentration of carbon dioxide becomes higher in the droplet and its back diffusion take place. Moles of dimethyl sulfoxide are continuous decreasing in the droplet. Fig. 3-Fig. 6 show moles of carbon dioxide in the droplet with respect to time, moles of dimethyl sulfoxide in the droplet with respect to time, mole fraction of carbon dioxide in droplet with respect to time.

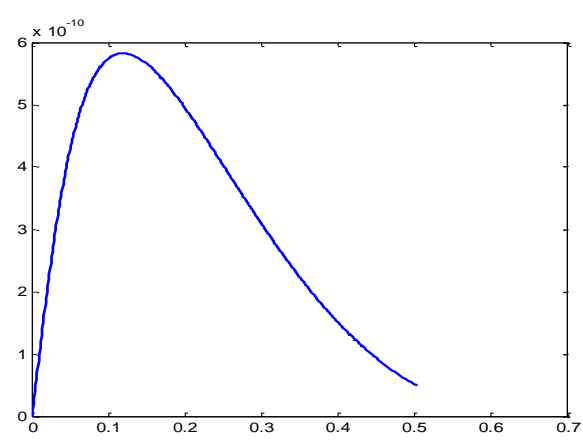

Fig. 3. moles of carbon dioxide v/s time $T=313 \mathrm{~K}, P=80 \mathrm{bar}, d_{n}=100$ $\mu \mathrm{m}, F=60 \mathrm{ml} / \mathrm{h}$

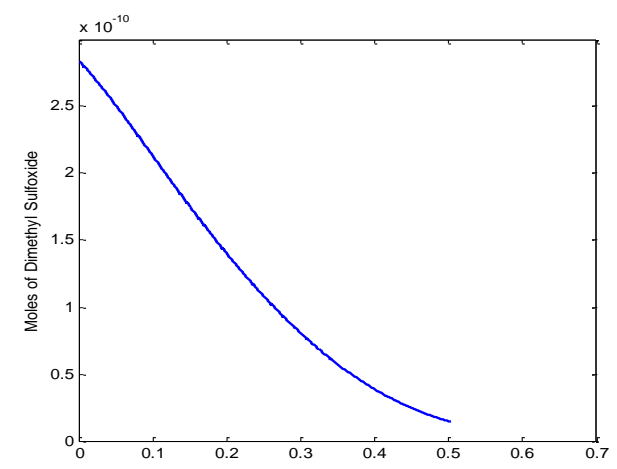

Fig. 4. Moles of dimethyl sulfoxide v/s time at $T=313 \mathrm{~K}, . P=80 \mathrm{bar}, d n=$ $100 \mu \mathrm{m}, F=60 \mathrm{ml} / \mathrm{h}$.

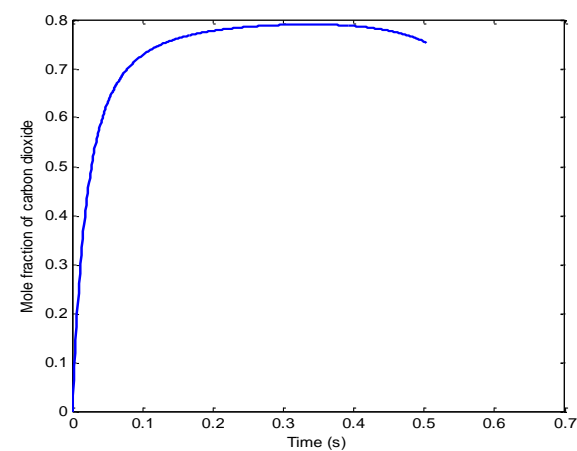

Fig. 5. Mole fraction of carbon dioxide v/s time at $T=313 \mathrm{~K}, P=80 \mathrm{bar}$, $d n=100 \mu \mathrm{m}, F=60 \mathrm{ml} / \mathrm{h}$.

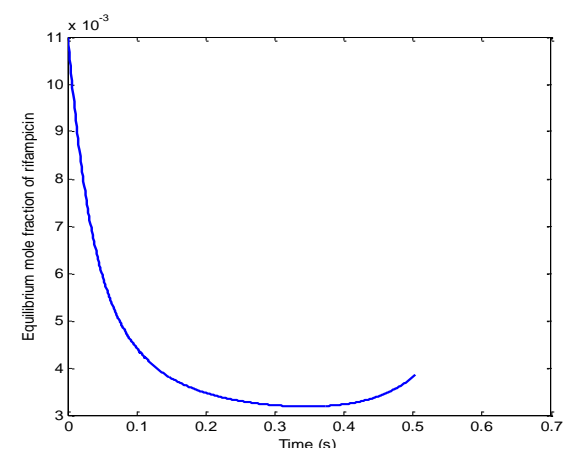

Fig. 6. Equilibrium mole fraction of rifampicin v/s time. 
Solute rifampicin is insoluble in carbon dioxide hence diffusion of carbon dioxide in dimethyl sulfoxiderifampicin mixture decreases the solubility of rifampicin in the droplet. As mole fraction of carbon dioxide increases in the droplet, equilibrium mole fraction of rifampicin decreases in droplet which is shown in Fig. 6.

\section{EFFeCt OF Flow RAte, Nozzle Diameter, PREssure AND TEMPERATURE}

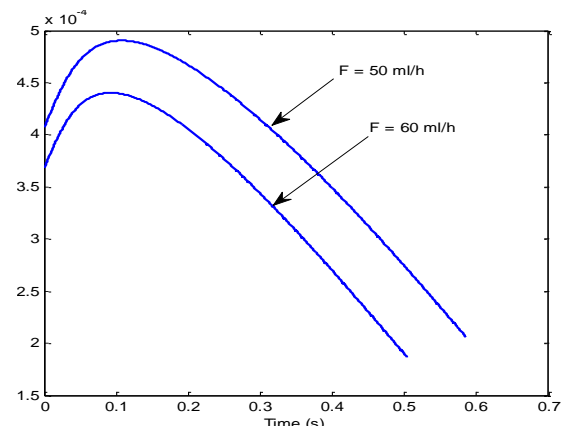

Fig. 7. Droplet diameter v/s time at two different flow rates.

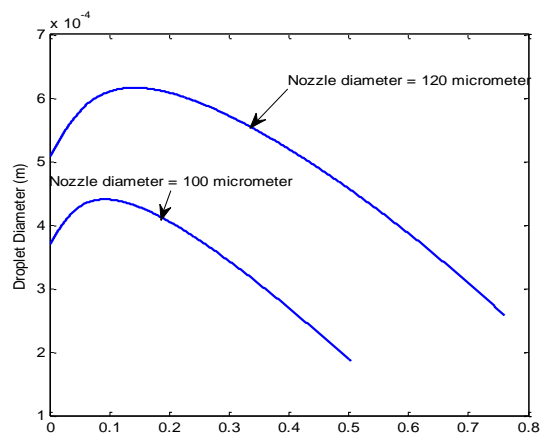

Fig. 8. Droplet diameter v/s time at two different nozzle diameters.

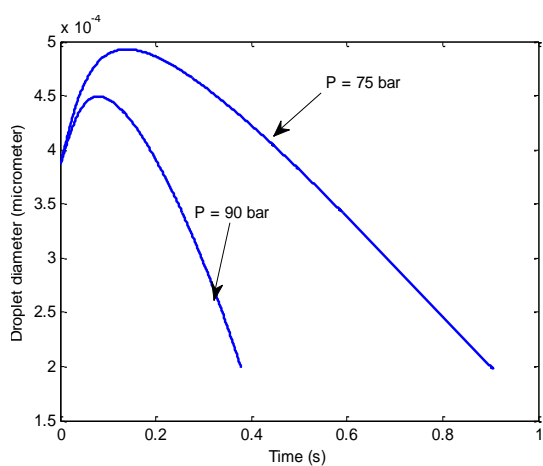

Fig. 8. Droplet diameter v/s time at two different pressure

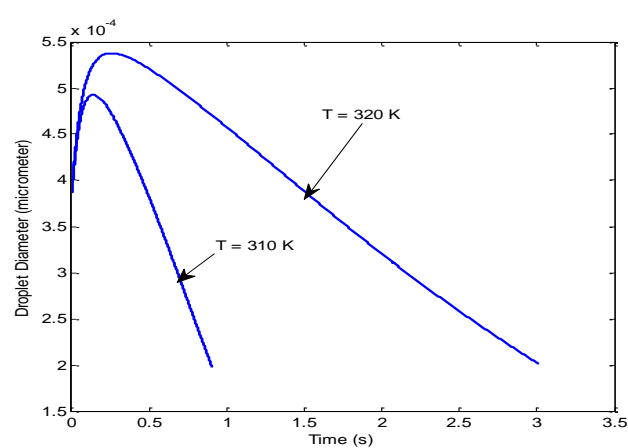

Fig. 9. Droplet diameter v/s time at two different temperatures.

Process parameter such flow rate, nozzle diameter,
Pressure and Temperature have a significant role on mass transfer between droplet and supercritical carbon dioxide. In this work, effect of these parameters have been studied and shown in Fig. 7-Fig. 9. This has observed that change in flow rate and nozzle diameter produced droplet of different diameter while pressure and temperature have little effect on initial droplet diameter.

\section{CONCLUSION}

Mass Transfer between droplet and supercritical carbon dioxide has been studied in this work and it is found that number of moles of a moving droplet increase first and then decreases. This is due to mass transfer of carbon dioxide from supercritical medium into droplet and then back diffusion from the droplet. Number of moles of dimethyl sulfoxide is decreasing continuously. Effects of flow rate, nozzle diameter, pressure and temperature have been studied. Higher flow rate leads to smaller droplet while larger nozzle diameter produces bigger droplet. Increasing the pressure in supercritical antisolvent process lead to fast evaporation of solvent while increasing the temperature slows the evaporation of solvent. Therefore higher flow rate, smaller nozzle diameter, higher pressure and lower temperature are the suitable to increase the evaporation of dimethyl sulfoxide and subsequently for lower residence time of moving droplet in the precipitator.

\section{REFERENCES}

[1] Y. H. Kim and K. S. Shing, "Supercritical fluid-micronized ipratropium bromide for pulmonary drug delivery," Powder Technology, vol. 182, pp. 25-32, 2008

[2] J. Fages, H. Lochard, J. Letourneau, M. Sauceau, and E. Rodier, "Particle generation for pharmaceutical applications using supercritical fluid technology," Powder Technology, vol. 141, pp. 219-226, 2004.

[3] V. Majerik, G. Charbit, E. Badens, G. Horvath, L. Szokonya, N. Bosc, and E. Teillaud, "Bioavailability enhancement of an active substance by supercritical antisolvent precipitatioin," J. of Supercritical Fluids, vol. 40, pp. 101-110, 2007

[4] J. Jung, and M. Perrut, "Particle design using supercritical fluids: Literature and Patent survey," J. of Supercritical Fluid, vol. 20, pp. 179-219, 2001.

[5] I. Kikic, N. D. Zordi, M. Moneghini, and D. Solinas, "Solubility estimation of drugs in ternary systems of interest for the antisolvent precipitation processes," J. of Supercritical Fluids, vol. 55, pp. 616622, 2010I.

[6] E. Reverchon, G. D. Porta, and P. Pallado, "Supercritical antisolvent precipitation of salbutamol microparticles," Powder Technology, vol. 114, pp. 17-22, 2001.

[7] I. Kikic, P. Alessi, F. Eva, M. Moneghini, and B. Perissutti, "Supercritical antisolvent precipitation of atenolol: The influence of the organic solvent and of the processing approach," $J$. of Supercritical Fluids, vol. 38, pp. 434-441, 2006.

[8] E. Franceschi, M. H. Kunita, M. V. Tres, A. F. Rubira, E. C. Muniz, M. L. Corazza, C. Dariva, S. R. S. Ferreira, and J. V. Oliveira, "Phase behavior and process parameters effects on the characteristic of precipitated theophylline using carbon dioxide as antisolvent," $J$. of Supercritical Fluids, vol. 44, pp. 8-20, 2008.

[9] E. Reverchon, and I. D. Marco, "Supercritical antisolvent micronization of cefonicid: thermodynamic interpretation of results," J. of Supercritical Fluids, vol. 31, pp. 207-215, 2004

[10] Y. Tien, C. Su, L. Lien, and Y. Chen, "Recrystallization of erlotinib hydrochloride and fulvestrant using supercritical antisolvent process," J. of Supercritical Fluids, vol. 55, pp. 292-299, 2010.

[11] A. Tenorio, M. D. Gordillo, C. Pereyra, and E. J. M. Ossa, "Controlled submicro particle formation of ampicillin by supercritical antisolvent precipitation," J. of Supercritical Fluids, vol. 40, pp. 308 316,2007 
[12] M. Kim, S. Lee, J. Park, J. Woo, and S. Hwang, "Micronization of cilostazol using supercritical antisolvent (SAS) process: Effect of process parameters," Powder Technology, vol. 177, pp. 64-70, 2007.

[13] I. D. Marco and E. Reverchon, "Supercritical antisolven micronization of cyclodextrin," Powder Technology, vol. 183, pp. 239-246, 2008

[14] E. Reverchon, R. Adami, G. Caputo, and I. D. Marco, "Spherical microparticles production by supercritical antisolvent precipitation: Interpretation of results," J. of Supercritical Fluids, vol. 47, pp. 70-84, 2008.

[15] E. Reverchon, G. D. Porta, I. D. Rosa, P. Subra, and D. Letourneur, "Supercritical antisolvent micronization of some biopolymers," J. of Supercritical Fluids, vol. 18, pp. 239-245, 2010.

[16] S. Chang, M. Lee, and H. Lin, "Role of phase behavior in micronization of lysozyme via a supercritical anti-solvent process," Chemical Engineering Journal, vol. 139, pp. 416-425, 2008

[17] M. Rossmann, A. Braeuer, S. Dowy, T. G. Gallinger, A. Leipertz, and E. Schluecker, "Solute solubility as criterion for the appearance of amorphous particle precipitation or crystallization in the supercritical antisolvent (SAS) process," J. of Supercritical Fluid, vol. 66, pp. 350-358, 2012

[18] F. E. Wubbolts, O. S. L. Bruinsma, and G. M. V. Rosmalen, "Measurement and modeling of the solubility of solids in mixtures of common solvents and compressed gases," J. of Supercritical Fluids, vol. 32, pp. 79-87, 2004.

[19] Y. P. Diego, F. E. Wubbolts, and P. J. Jansens, "Modelling mass transfer in the PCA process using the Maxwell-Stefan approach," J. of Supercritical Fluids, vol. 37, pp. 53-62, 2006.
[20] R. Kumar, H. Mahalingam, and "K. Tiwari, Modeling of droplet composition in supercritical antisolvent process: part A," International Journal of Chemical Engineering and Applications, vol. 3, no. 6, pp. 456-460, 2012.

[21] M. Mukhopadhyay and S. V. Dalvi, "Partial molar volume fraction of solvent in binary ( $\mathrm{CO} 2$ - solvent) solution for solid solubility predictions," J. of Supercritical Fluids, vol. 29, pp. 221-230, 2004.

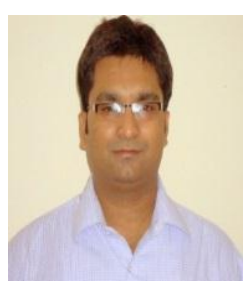

Rahul Kumar was born at Bijnor (U. P.) in India He completed his B.Tech degree in chemical engineering from Bundelkhand Institute of Engineering \& Technology Jhansi and M.Tech degree from Indian Institute of Technology Bombay. He is currently working as a senior lecturer at Jaypee University of Engineering \& Technology. He has presented a research article titled "Droplet dynamics in supercritical antisolven micronization process" at 4th International Conference on Chemical, Biological, and environmental Engineering held in Phuket, Thailand on September 1-2, 2012. His current interests are crystallization, vapor-liquid equilibria, and supercritical fluids.

H. Mahalingam is currently an associate professor and the head of Department of Chemical Engineering, Jaypee University of Engineering \& Technology, Guna, M.P., India. He secured his Ph.D degree in chemical engineering from the National University of Singapore and was a postdoctoral researcher in France/Singapore. He obtained his B.Tech. and M.Tech. degrees in chemical engineering from Anna University, Chennai in 1994 \& 1996 and has about 14 years of teaching \& research experience. 